\title{
Competitive Routing on a variant of Delaunay Triangulation
}

\author{
Virendra Singh Rathore \\ Department of Computer Science \\ Lakehead University \\ Thunder Bay, Canada \\ vrathore@lakeheadu.ca
}

\begin{abstract}
The concept of Delaunay triangulation is thought to be currently one of the best implementations in the sampling arena, whether it be technical or a non technical domain. Considering the network congestions which cause a competitive routing in any given area of the network, Delaunay triangulation has come to be proven as a good, if not the best, remedy to solve the mentioned problem. Dr. Prosenjit Bose presented a good argument back in November 2011 where he proved that connecting the nodes of any given network using the concepts of Delaunay Triangulation gave the best path between nodes, taking the least amount of time for the communication and reducing the competitive routing in the network by reducing the spanning ratio and path length by almost 5/sqrt(3). Here in this study we use the concepts of the Delaunay Triangulation to design a Java application which analyses given a set of random nodes in a plane, it connects each of them with the use of Delaunay Triangulation so that the nodes have the best path to communicate with each other.
\end{abstract}

Keywords- Delaunay Triangulation, graph theory, Euclidean Distance, Spanning ratio, Competitive Routing, Java, Eclipse IDE

\section{INTRODUCTION}

The study here covers the major theory which comprises the foundation and base of the Delaunay Triangulation. The basics include the graph theory which is the main component of the design and processing of the Delaunay triangulation. The presented topic majorly covers two important concepts; namely 'Competitive Routing' and 'Delaunay Triangulation'. Here in our study, we explore one variant of the of the several applications of the Delaunay Triangulation, that is how the Delaunay Triangulation can be used to solve the problem caused by Competitive Routing on a computer network. In this study we basically aim to design a platform where we see how nodes in the given plane, which represent network components of various kinds, are placed and connected in such a way that they form a proper Delaunay triangulation connection amongst each other and hence are eligible to provide the least spanning ratio obtained by using various other connecting techniques. As the theory ends we see how we design an application program using Java in Eclipse IDE and gradually see the triangulation getting designed in action.

\section{GRAPH THEORY}

\section{A. Graphs, the key components}

As one starts to study what Delaunay Triangulation is, one realizes that it is all about Graphs and fundamentals related to graphs. The Delaunay triangulation was introduced to solve the problems arising in interconnected nodes on a plane in the first place. Therefore to begin the study of Delaunay Triangulation, one has to familiarize himself with the concepts of graphs and all the terms and concepts related to graphs.

Graph: "In mathematics, and more specifically in graph theory, a graph is a structure amounting to a set of objects in which some pairs of the objects are in some sense "related". The objects correspond to mathematical abstractions called vertices (also called nodes or points) and each of the related pairs of vertices is called an edge. Typically, a graph is depicted in diagrammatic form as a set of dots for the vertices, joined by lines or curves for the edges. Graphs are one of the objects of study in discrete mathematics." (Wikipedia)

It can be clearly understood from the above given definition that the nodes represent 'related' or 'similar' sets of objects. When we talk about Computer Networks, there are complex networks interconnecting several devices and machines across a given area. So in order to simplify and understand the Computer Network, we use 'Graphs'. Using graphs is the most popular and elementary way to analyze, study and apply alterations to composite networks. There are various types of graphs that can be described which are used to depict different types of networks used across the globe. Here we will discuss the ones which are significant to study the application of the Delaunay Triangulation.

Simple Graph: A simple graph, also called a strict graph, is an unweighted, undirected graph containing no graph loops or multiple edges. 


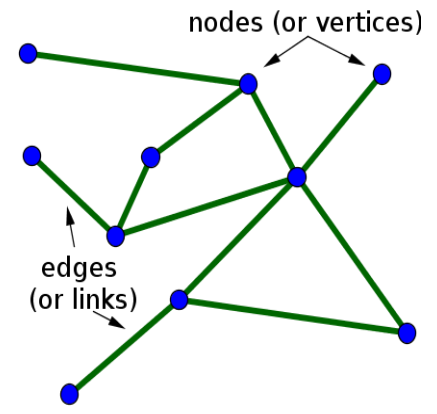

Weighted Graph: A weighted graph is a graph in which each branch/edge is given a numerical weight. This weight can represent anything from node one to two; for example their distance, time taken to send data between those nodes, etc.

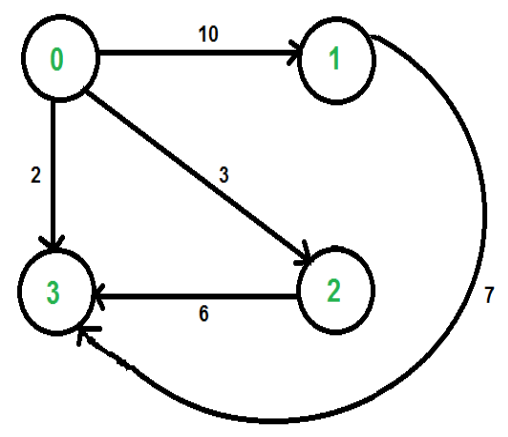

Directed/ Undirected Graph: A directed graph is graph in which a set of nodes that are connected together, where all the edges are directed from one vertex to another. A directed graph is sometimes called a digraph or a directed network. In an undirected graph, the nodes that are connected together have no particular direction. All the edges are bidirectional.

Spanning Tree, Graph: A spanning tree is a subset of Graph $G$, which has all the vertices covered with minimum possible number of edges. Hence, a spanning tree does not have cycles and it cannot be disconnected. By this definition, we can draw a conclusion that every connected and undirected Graph
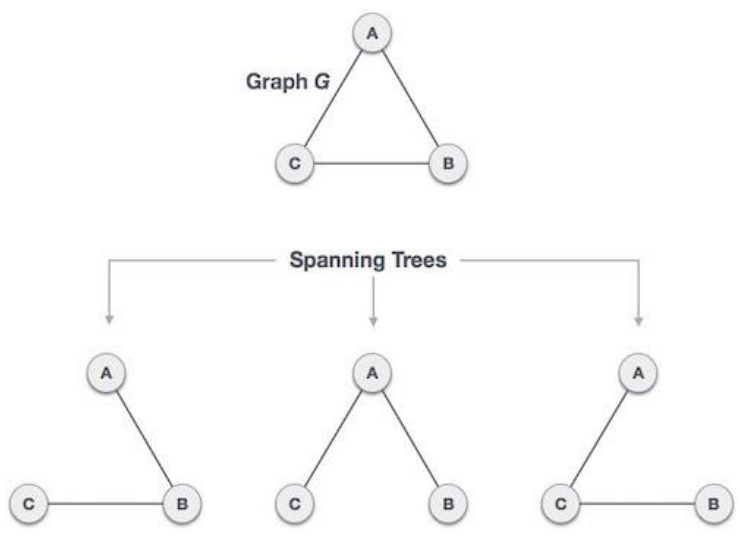

$\mathrm{G}$ has at least one spanning tree.
Euclidean Graph: A Euclidean graph is a graph in which the vertices represent points in the plane, and the edges are assigned lengths equal to the Euclidean distance (linear distance) between those points.

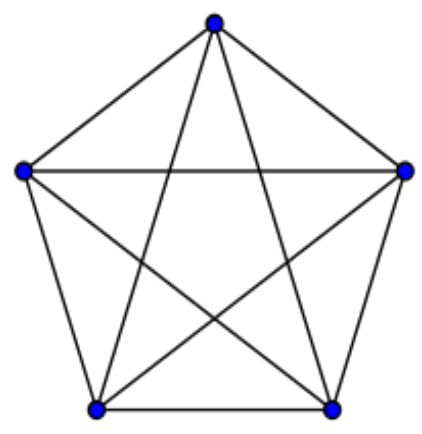

\section{B. Routing and Competitive Routing}

Now as we talk about graphs which represent a network, we need to specify what routing is. Routing is the process of selecting a path for traffic in a network, or between or across multiple networks. Whenever a node has to send data/message to another node in the network, it chooses the best route that will send the data in the shortest amount of time/path. Every node (which can be a station/device/router) has its own routing algorithm to find out the best and shortest route to all other nodes in the network. This process of finding and using the best routes to transfer data amongst various nodes in the network in called routing. Routing Algorithms improve the performance of the network by implementing the best routing protocols.

But gradually what happens is, although these Routing Algorithms are meant to improve the overall performance of the network, a contradiction is observed when the traffic in the system is high. We consider a communication network shared by several selfish users. Each user seeks to optimize its own performance by controlling the routing of its given flow demand, giving rise to a non-cooperative game. This result in the degradation of even the best possible routes from point one to point two. Proper recognition and solution for such problems is then required to maintain the best possible performance of the system. One such solution is seen when we apply the concept of Delaunay Triangulation when connecting the nodes in the network.

The diagram below depicts an example where we can discuss how a competitive routing scenario can occur in a working network and degrade the performance of the system. Consider the node ' $s$ ' and node ' $t$ ', where 
node ' $s$ ' sends message $M$ to node ' $t$ ' via node ' $v$ '. Each edge is weighed with a capacity (c) and a delay factor (d). Other nodes also use the same path via node ' $v$ ' to send messages/data to node ' $t$ '. The reason for that is there is no delay in the edge between node ' $\mathrm{v}$ ' and node ' $t$ ', so all algorithms choose the $(\mathrm{c}=1, \mathrm{~d}=0)$ path to node ' $t$ '. But gradually when the traffic rises, the load becomes more than the capacity and hence this competition of choosing the best path to node ' $t$ ' degrades that route. Soon the system realizes that the path is no longer the best one, so gradually the algorithms decide that the other path $(\mathrm{c}=1, \mathrm{~d}=1)$ becomes the best path to node' $t$ ' despite having a delay $d=1$. The competition still continues to occur everywhere in the system and hence delay and congestion is observed.

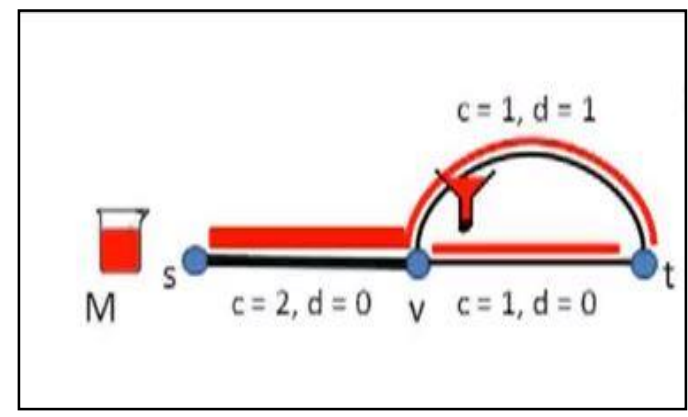

C-competitive route: The sequence of adjacent nodes visited by a routing algorithm on a graph $\mathrm{G}$ between given source and target nodes $\mathrm{s}$ and $\mathrm{t}$ is a ccompetitive route if its length in $G$ is at most $c$ times the length of the shortest path from $s$ to $t$ in $G$.

Here we present different c-competitive online routing algorithms on Delaunay triangulation of an arbitrary given set of points in the plane like

1. $4 \pi \vee 3 \approx 21.766$ - competitive online routing algorithm

2. $9 * 1.998 \approx 17.982$ - competitive online routing algorithm

This is the improvement of competitive ratio on Delaunay triangulations from the previous best of $9(1$ $+\sqrt{ } 5) \pi / 2 \approx 45.749$ - competitive online routing algorithm.

We also present a competitive online routing algorithm for Delaunay Triangulations of point of convex position and that is $(11+3 \sqrt{ } 2) / 2 \approx 7.621$-competitive online routing algorithm.

We can design online routing algorithms with good competitive ratios by focusing on specific local triangles in Delaunay Triangulation of points $\mathrm{P}$ i.e. DT $(\mathrm{P})$, to reduce the search space of candidate vertices to which we need to forward our message and by exploiting the geometric properties of Delaunay Triangulation. The search space is restricted by focusing on two specific paths that lie respectively above and below the line segment from $\mathrm{s}$ to $\mathrm{t}$, where $\mathrm{s}$ and $t$ denote the respective source and target nodes in DT(P). In the above (Figure a), if you consider the ordered sequence of triangles that intersect the line segment st, each triangle in this sequence has at least one edge whose interior is either completely above or completely below the line segment st.

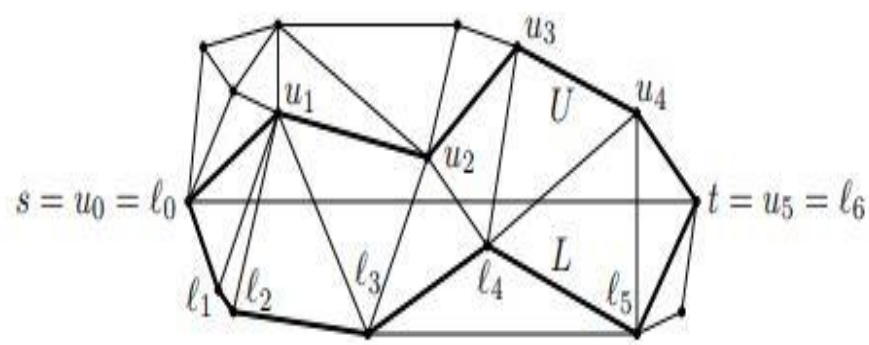

\section{Pseudo code:}

1. Input()

2. Plot points(Graph G)

\{

Plot the graph $\mathrm{G}$ with the set of vertices $\mathrm{V}$ and edges $\mathrm{E}$ and the given weights $\mathrm{W}$

$$
\text { \} }
$$

3. Delaunay Triangulation(Graph G)

$$
\{
$$

Re-arrange the edges between points so that there is no other point in $\mathrm{P}$ is inside the circle.

4. Transfer packets within any two given vertices finding the shortest path using Delaunay triangulation.

5. Display the sub-graph used to find the shortest distance between two points.

\section{Stop().}

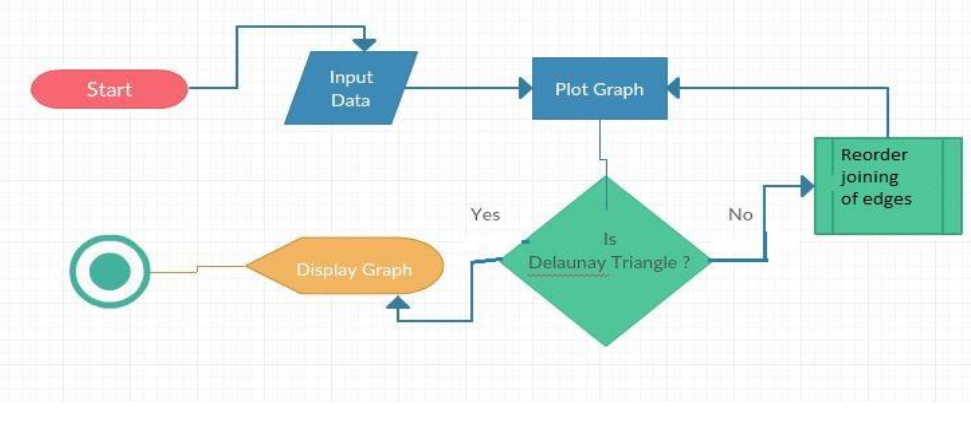




\section{IMPLEMENTATION}

\section{Design:}

1. We are using Java to implement.

2. We will show the simulation of Delaunay Triangulation.

3. We will plot some points as vertices and show the difference when we use the Delaunay Triangulation in computing the competitive path in a network and how it is efficient from other usual methods.

\section{Tools and methodology:}

Java is one of the most common programming languages that is used in almost more than 6 million devices across the world. "Java is a general-purpose computer-programming language that is concurrent, class-based, object oriented, and specifically designed to have as few implementation dependencies as possible. It is intended to let application developers 'write once, run anywhere' meaning that compiled Java code can run on all platforms that support Java without the recompilation"-Wikipedia. This ease where Java gives us a platform independent applications has a plus point too. Once we make an application on any particular platform, it can be run on multiple other platforms that has Java Environment Variables (JRE).

IDE: Although Java can be implemented on various available IDE's, to create an interface where the Delaunay Triangulation can be calculated, we are using the Eclipse Mars IDE. This IDE provides various tools and options to make the work more efficient. The following steps are taken to design the intended system;

\section{A. Designing the Frameset}

Being specific on the toolkit, we have used the Abstract Window Toolkit (AWT) to simulate the complete process of triangulation. The Java AWT contains classes for creating user interfaces and for painting graphics and images. A container in AWT is a component that can contain components and other containers. A container can also have a layout manager that controls the visual placement of components in the container. The AWT package contains several layout manager classes and an interface for building your own layout manager. Each component object is limited in its maximum size and its location because the values are stored as an integer as said by the documentation given by Oracle. The AWT class in java has provided support for Graphics2D class which was used to plot the points on a given canvas. The important feature of AWT class here was to handle events. Events in computer programming, is a programming paradigm in which the flow of the program is determined by events such as user action such as mouse clicks and key presses or sensor outputs or messages from other programs. Event-driven programming is the dominant paradigm used in graphical user interfaces and other applications that are centered on performing certain actions in response to user input. Though the AWT class has many features the advance components in the user interface were supported by the SWING API in java. Java Swing API is actually based on the AWT class but has advanced components to ease the GUI Development such as JButton, JScrollPane, JTextArea, Jframe, JMenu, and JFileChooser.

\section{B. Simulation}

- To simulate the formation of Delaunay triangulation we have made a user interface which accepts the set of points as an input file and extracts the set of points and plots them on the canvas.

- As the interface buttons are clicked to calculate the Delaunay Triangulation on the randomly plotted points on the plane, the code executes the program code and analyses the connections on the given set of points so as to plot the nest connection amongst the points.

- The points as shown in the final plotted 'graph' on the screen shows the proper connection of the nodes based on the Delaunay Triangulation rules as described in the program code in Java.

\section{Results}

After the coding process is completed, we end up having a frameset where we can use the mouse cursor to click and create random or prespecified set of points which represent nodes in a Computer Network. As soon as the 'Calculate Triangulation' button is pressed, the algorithm defined in the program code executes and processes the given set of points, the points are examined so as not to fall in any of the circles formed by the 3 nodes as explained earlier. If it does it is detected as a bad triangulation and the connections are changed. Finally we get the perfectly interconnected nodes based on the concepts of the Delaunay Triangulation. This connection shows how the nodes should be connected if they were at all a part of the network, and hence they undergo the least amount of delay caused by congestion and/or competitive routing. If new nodes are also introduced in the system after triangulation, the system is efficient to recalculate the entire system 
once again and give the next best option available for the Delaunay Triangulation Solution.

Additionally, the program provides a feature to save and retrieve a set of previously used points. If the system is being used to analyse an actual required set of points, for example airline route analysing, the set of points given in the plane of this program can be saved and used in later future for reference purposes. The following screenshots depict the program console;

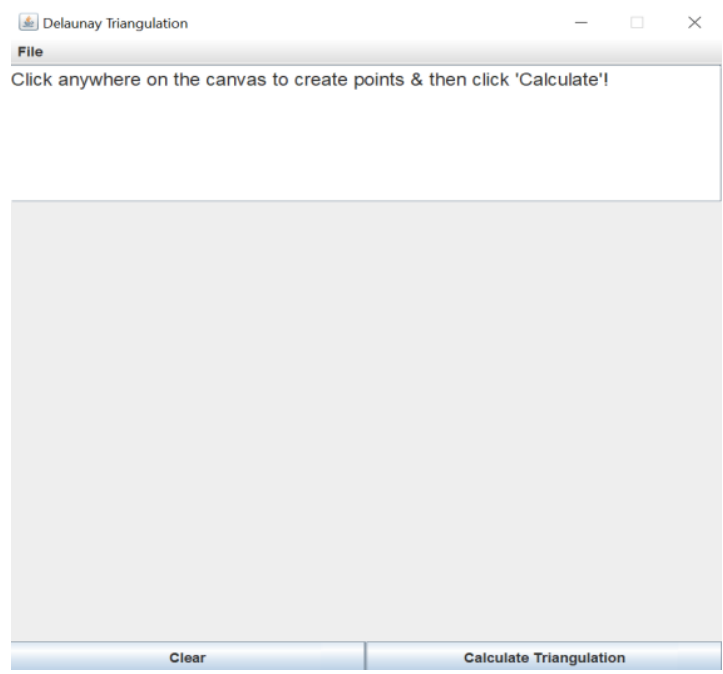

The Main Frame Screen

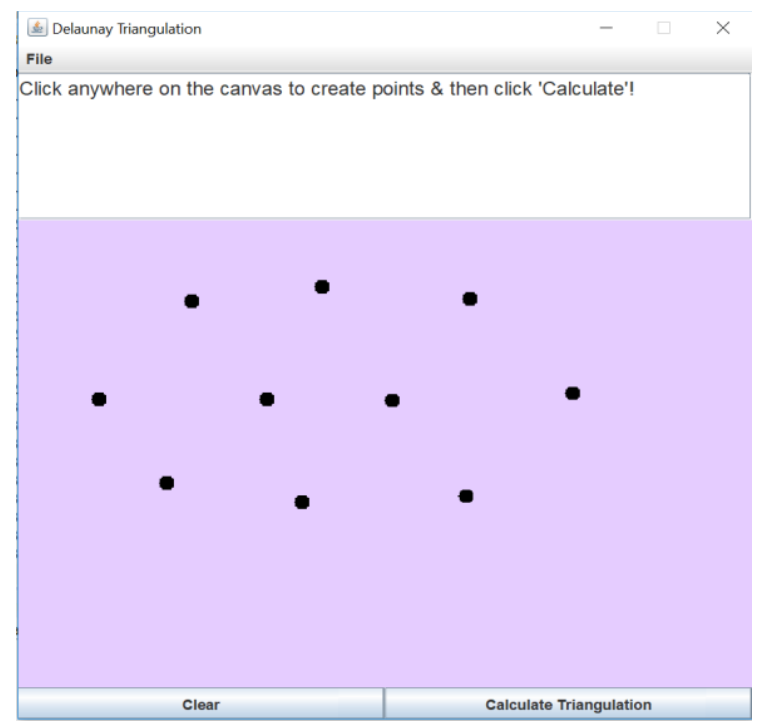

Random nodes plotted on screen frame

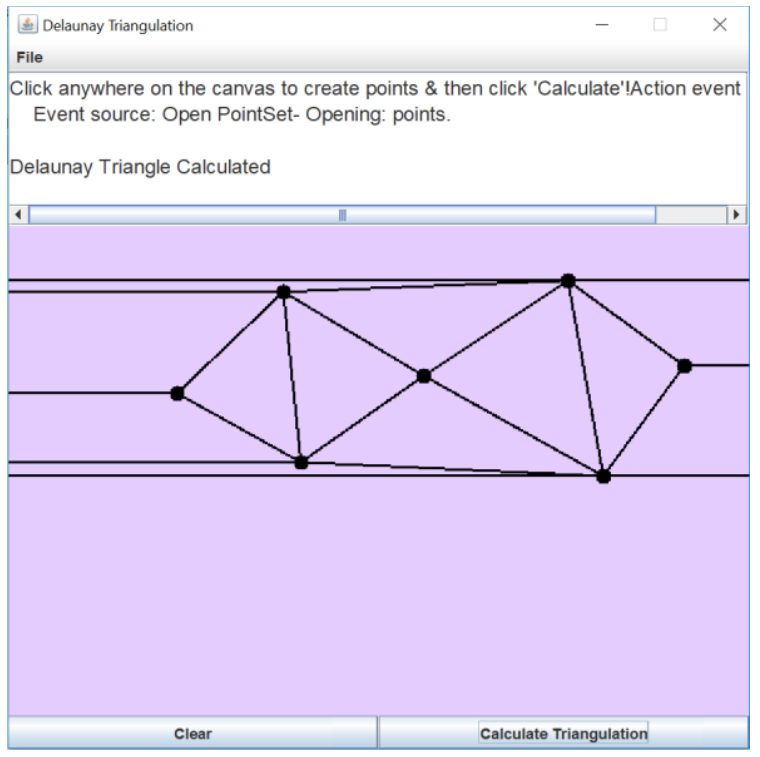

Connections after Triangualtion

\section{CONCLUSION}

We find that given any set of points in a plane, the nodes can be taken into as a set of vertices and edges of a graph and Delaunay Triangulation can be geometrically applied on the set of nodes so as to find the best triangulated connections between them.

After the system has been designed, now future research can be implemented by running the system under a simulation where data packets can be sent to and fro between various nodes and then the calculation can be done that Delaunay Triangulation does indeed cause the network connections to be more efficient than any other connection and congestion and competitive routing can be highly reduced as described earlier in the report.

While this said, Delaunay Triangulation is still a very new concept that has been applied to Computer Network and other Computer System such as designing, etc. There can be various modulations that can be made in coming near future that may lead to the enhancement of the currently proposed system. Hence the research in the area, though concluded for now, remains to be an area of further constructive development.

\section{ACKNOWLEDGMENT}

We would like to thank Dr. Jinan Fiaidhi for her immense help and support, useful discussions and valuable recommendations.

Hence we have seen what the Delaunay Triangulation is based on, the basics, its usage in computer networks and to to design an application using Java to implement the functionality and see the triangulation happen in real time. 


\section{REFERENCES}

[1] http://www.cs.tufts.edu/t/colloquia/current/?event=774

Competitive Routing ON A VARIANT OF the DELAUNAY TRIANGULATION

November 10, 2011

2:50 pm - 4:00 pm

Halligan 111

Speaker: Jit Bose, Carleton University

Host: Diane Souvaine

\section{A. Abstract}

A subgraph $\mathrm{H}$ of a weighted graph $\mathrm{G}$ is a t-spanner of $\mathrm{G}$ provided that for every edge $\mathrm{xy}$ in $\mathrm{G}$, the weight of the shortest path between $\mathrm{x}$ and $\mathrm{y}$ in $\mathrm{H}$ is at most $\mathrm{t}$ times the weight of xy. It is known that the Delaunay triangulation of a point set $\mathrm{P}$ (where the empty region is an equilateral triangle) is a 2-spanner of the complete Euclidean graph. We present a new and simple proof of this spanning ratio that allows us to route competitively on this graph. Specifically, we present a deterministic local routing scheme that is guaranteed to find a short path between any pair of vertices in this Delaunay triangulation. We guarantee that the length of the path is at most 5/sqrt(3) times the Euclidean distance between the pair of vertices.

Moreover, we show that no local routing scheme can achieve a better competitive spanning ratio thereby implying that our routing scheme is optimal. This is somewhat surprising since the spanning ratio is 2 .

Bio:

[1] Prosenjit Bose received his B. Math (1990) and M. Math (1991) in Computer Science and Combinatorics from the University of Waterloo, Canada. He completed his Ph.D. (1994) in Computer Science at McGill University, Canada, where he received the D.W. Ambridge Award as the outstanding Ph.D. graduate. Currently, he is a full professor at the School of Computer Science and the associate dean for research in the Faculty of Science at Carleton University, Canada. He has received several research and teaching awards. His main research area is applied geometric computing. He has published over 200 journal and conference papers in this area.

[2] https://en.wikipedia.org/wiki/Delaunay triangulation

[3] https://www.semanticscholar.org/paper/Optimal-localrouting-on-Delaunay-triangulations-by-BoseFagerberg/e08c3e1c5df4f0dd5b2843b45c1707f6c4c0263c [4]https://docs.oracle.com/javase/7/docs/api/java/awt/packa ge-summary.html\#package_description 